\title{
EFICIENCIA TERMINAL EN PROGRAMAS DE LICENCIATURA, URACCAN BILWI 1995 -2005
}

\author{
Argentina García Solórzano ${ }^{[1]}$
}

Earl Tom ${ }^{[2]}$

\section{Resumen}

Se estudió la Eficiencia Terminal en el período 1995 - 2005 en la URACCAN Bilwi, describiéndose algunos factores que inciden en el bajo porcentaje de titulación. La investigación realizada es cuantitativa. Tiene un carácter documental y descriptivo. Las técnicas aplicadas fueron la encuesta a los egresados y la revisión documental por medio de las estadísticas de registro académico.

Entre los hallazgos se destaca que en la URACCAN Bilwi, de 1628 estudiantes que ingresaron durante el período estudiado se graduaron 752 , lo que significa el 38\% de índice de Eficiencia Terminal a pesar de los planes de seguimiento y atención implementados para agilizar los procesos de graduación. El estudio aporta el índice de eficiencia alcanzado, factores por los cuales los egresados no han logrado la Eficiencia Terminal, cómo se han facilitado las nuevas estrategias, así la propuesta de un conjunto de medidas correctivas para mejorar los índices de Eficiencia Terminal.

Palabras clave: Índice de graduación, Recinto Bilwi, estrategias y medidas correctivas.

\section{Summary}

Graduation rate was studied in the period $1995-2005$ in URACCAN Bilwi, describing some factors that affect the low percentage of degree. The research is quantitative. It has a documentary and descriptive character. The techniques were applied through surveys to graduates and document review through academic registry statistics.

Among the findings we emphasize that in the URACCAN-Bilwi, from a total of 1,628 students admitted during the period that was analyzed, 752 graduated, which means $38 \%$ of graduation rate, despite monitoring plans that were implemented

\footnotetext{
[1] Máster en Estudios Interdisciplinarios, Secretaria Académica Recinto Bilwi, argentina_garcia@yahoo.com

[2] Máster en .Desarrollo con Identidad con mención en Gobernabilidad, territorialidad y manejo de bosque. Co-investigador. earltom202@yahoo.com
} 
to maximize the graduation processes. The study contributed to the efficiency rate achieved, factors why graduates have failed terminal efficiency, how we have facilitated new strategies as well as a proposal with a set of corrective measures to improve terminal efficiency rates.

Keywords: Graduation rate, Bilwi Campus, strategies and corrective measures.

\section{Introducción}

La URACCAN se define como: "Universidad comunitaria intercultural de los pueblos indígenas y comunidades étnicas". El concepto de universidad comunitaria, avalado por el Consejo Regional Autónomo, se inscribe en el marco del reconocimiento y ejercicio de los derechos humanos y colectivos que los pueblos indígenas y afrodescendientes que han reivindicado en el proceso de construcción de las Regiones Autónomas multiétnicas.

En la URACCAN, la Eficiencia Terminal se valora como criterio para evaluar el funcionamiento de la Educación Superior. Es un indicador que da cuenta de los logros obtenidos por las instituciones educativas, en cierta medida refleja la calidad de los programas ofertados y de las instituciones. Se distingue entonces, como uno de los indicadores que permiten reconocer los productos alcanzados por las instituciones educativas en diferentes niveles (Mendoza, 2003).

En el caso del Recinto Bilwi la Eficiencia Terminal promedio es de $38.16 \%$, tomando en cuenta como criterio a los nuevos ingresos por carrera y los graduados por carrera en el período 1995-2005. Se conoce que en el recinto Bilwi algunos estudiantes solamente egresan y no se gradúan en el período que debieran hacerlo.

La Eficiencia Terminal es un criterio de calidad para la acreditación institucional y en este sentido se ha planteado conocer el índice de Eficiencia Terminal de los programas de licenciatura de URACCAN de las generaciones 1995-2005, los factores que inciden por los cuales el estudiantado no culmina sus estudios en tiempo y forma, porcentajes de estudiantes que no se graduaron y cuánto tiempo duraron; asimismo, las estrategias que está siguiendo la universidad para que sus egresados logren su Eficiencia Terminal.

Este estudio pretende contribuir a elevar los índices de Eficiencia Terminal en este campus universitario y por ende, en toda la Universidad. 


\section{Marco Teórico}

\section{Eficiencia Terminal}

"La Eficiencia Académica Terminal (EAT) puede ser considerada como un atributo que expresa la efectividad y calidad educativa del proceso de enseñanza-aprendizaje de una institución de educación estimada en un tiempo determinado y situaciones dadas". Echemendía. M; García. T; Torres. E; Sánchez. D; Rodríguez. Y; y Torres. A. (2008. Para Cuellar Saavedra \& Bolívar Espinoza, la Eficiencia Terminal se define como: "El grado en que se logra que los alumnos que ingresan al sistema educativo avancen a lo largo de los grados que comprende el nivel educativo en la forma prevista"

La Eficiencia Terminal se ha definido desde diversas perspectivas como: la relación cuantitativa entre los alumnos que ingresan y los que egresan de una cohorte Huerta \& De Allende (1988) y Rangel Guerra (1979) la define como indicador que expresa la capacidad para lograr que quienes inician un nivel educativo determinado se gradúen satisfactoriamente en el mismo (Muñoz Izquierdo,1973).

La Eficiencia Terminal "se constituye, desde la perspectiva de la teoría de sistemas aplicada a la educación, en un indicador de la eficiencia interna con que funcionan y rinden las instituciones educativas, entendidas como sistemas" (Camarena R., Chávez A., \& Gómez J., s.f)

La Eficiencia Terminal se ve obstaculizada por distintos factores, así lo afirma Rojas (2009: 1): "la persistencia e incremento sostenido de la deserción universitaria puede ser, además, síntoma de una profunda crisis social en la valoración de la educación".) ANUIES (2000) plantea que las instituciones han reconocido que aún persisten problemas en los procedimientos de titulación, que no siempre están ligados a razones académicas; además, los que abandonan sus estudios lo hacen sin ninguna calificación reconocida, lo que implica un alto costo humano y financiero.

\section{Materiales y método}

La investigación realizada es cuantitativa y se realizó con fines académicos a fin de poder aportar conocimientos que ayuden a la toma de decisiones en la universidad en pro de elevar el porcentaje de Eficiencia Terminal logrado a la fecha. Tiene un carácter documental y descriptivo y las técnicas aplicadas fueron la encuesta a egresados/egresadas y la revisión documental. Se aborda la Eficiencia Terminal de 1628 estudiantes matriculados como primer ingreso; los 752 graduados y los 88 egresados del estudiantado que ha ingresado a URACCAN Recinto Bilwi desde 1995 hasta el 2005. Así mismo se documenta por medio de las estadísticas de la universidad, el año de ingreso, egreso y culminación de estudios del estudiantado. 
La Eficiencia Terminal se calculó como el coeficiente entre los titulados en el año " $\mathrm{t}$ " y la matrícula nueva de acuerdo a la duración promedio estipulada en los planes de estudio de cada una de las carreras seleccionadas. Esto es: $\mathrm{E}=\mathrm{T}(\mathrm{t}) / \mathrm{N}(\mathrm{t}-\mathrm{d}) \mathrm{En}$ donde: " $\mathrm{T}$ " corresponde a los titulados en el año ( $\mathrm{t}$ ) ; " $\mathrm{d}$ " es la duración promedio de las carreras en años según planes de estudio " $\mathrm{N}$ " ( $\mathrm{t}-\mathrm{d}$ ) es la matricula nueva en primer año para año correspondiente de acuerdo a la duración de la carrera.

\section{Resultados y discusión}

\section{Índices de Eficiencia Terminal 1995-2005}

Una de las perspectivas de cómo se aborda lo que es la Eficiencia Terminal según Huerta y De Allende (1988) es la relación cuantitativa entre los alumnos que ingresan y los que egresan de una cohorte. Con respecto al primer objetivo a continuación se detallan los hallazgos en cuanto a índice de Eficiencia Terminal.

Desde la apertura del Recinto URACCAN Bilwi hasta la fecha, se han ofertado veinticinco carreras de pre-grado para licenciaturas y una de ingeniería, se incluye el Técnico Superior que desde el año 2009 inició la carrera de Medicina Intercultural.

\section{Carreras que ha ofertado la universidad}

Para tener una idea de las áreas en que la URACCAN Bilwi ha graduado a profesionales costeños desde que dio apertura, se mencionan las siguientes: Informática Administrativa, Sociología con mención en Autonomía, Administración de Empresas con mención en Recursos Naturales, Banca y Finanzas, Administración Pública, Administración en Mercadeo, Medicina Intercultural, Ciencias de la Educación con mención en Pedagogía en Educación Primaria, Ciencias de la Educación con mención en Español, Ciencias de la Educación con mención en Biología, Ciencias de la Educación con mención en Historia, Ciencias de la Educación con mención en Geografía, Ciencias de la Educación con mención en Inglés, Educación Intercultural Bilingüe, Ciencias sociales con mención en Desarrollo Local, Comunicación Intercultural, Ingeniería Agroforestal, licenciaturas en Enfermería con mención en Epidemiología, Enfermería con mención en Materno Infantil y Enfermería con mención en Salud Mental.

Como Técnico Superior ha ofertado: Técnico Superior en Ciencias Naturales, Técnico Superior en Ciencias Sociales, Técnico Superior en Español, Técnico Superior en Informática, Técnico Superior en Enfermería, Técnico Superior en Desarrollo Municipal, Técnico Superior en Forestería y la Escuela de Liderazgo Preparatoria. 


\section{Relación cuantitativa entre ingreso y graduación 1995-2005}

Según datos del registro académico, se reporta que en el período de 1995 a 2005 ingresaron a la universidad 1628 alumnos que optaron a las carreras de: Ingeniería Agroforestal, Ciencias de la Educación, Enfermería, Informática Administrativa, Administración de Empresas, Sociología con mención en Autonomía, Comunicación Intercultural y Técnico Superior en Desarrollo Municipal y Forestal.

De 1995 al 2005 se graduó un total de 752 graduados/graduadas que representan el $38.16 \%$ de efectividad con relación a nuevos ingresos que optan por iniciar una carrera académica y los que llegan a la culminación de sus estudios hasta la graduación.

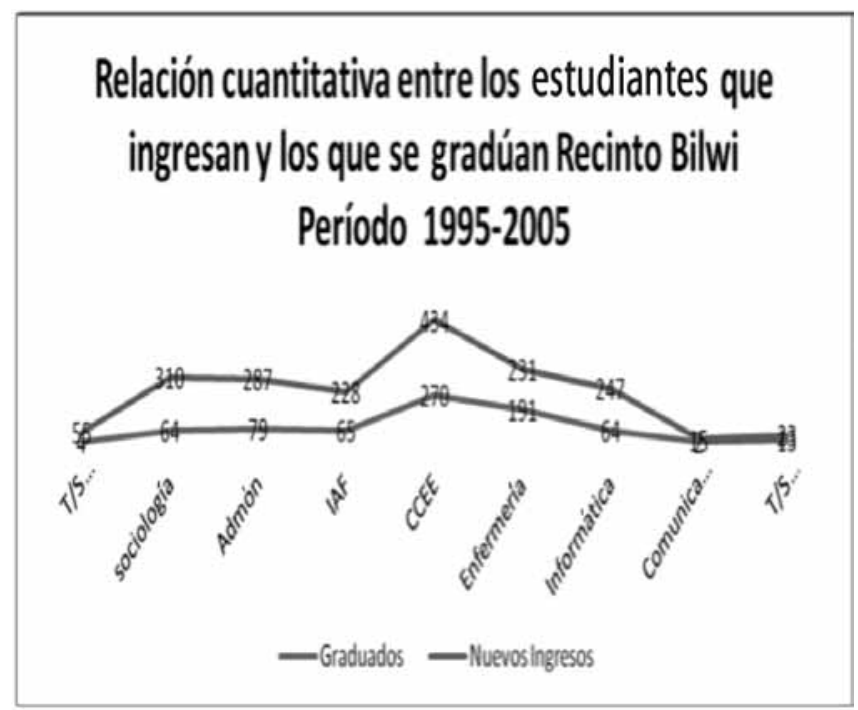

Gráfico No. 1. Eficiencia Terminal, 1995-2005.

La relación entre el número de alumnos que se inscriben por primera vez en una carrera profesional, formando a partir de este momento una determinada generación, y los de la misma generación que logran egresar, al haber acreditado todas las asignaturas correspondientes al currículo de cada carrera, en los tiempos estipulados por los diferentes planes de estudio (Camarena, Chávez, Gómez, 1983:7).

Conforme el cuadro siguiente se puede observar que ninguna de las carreras llegó al 90\% de Eficiencia Terminal.

La relación entre nuevo ingreso y graduados está bastante bajo y se llega de manera global al 38.16\%. Es llamativa la situación en cuanto a que hace reflexionar que hay que buscar mejores alternativas, mejores formas de atención a los egresados y egresadas, así a como está el índice de eficiencia es nocivo de cara a la acreditación institucional. 
Cuadro No. 1. Índices de Eficiencia Terminal

\begin{tabular}{|c|c|c|c|}
\hline Carrera & Graduados & Nuevos Ingreso & \% de Eficiencia \\
\hline T/S Forestal & 4 & 55 & 7.27 \\
\hline Sociología & 64 & 310 & 20.65 \\
\hline Admón. & 79 & 287 & 27.53 \\
\hline IAF & 65 & 228 & 28.51 \\
\hline Enfermería & 191 & 231 & 82.68 \\
\hline CCEE & 270 & 333 & 81.08 \\
\hline Informática & 64 & 247 & 25.91 \\
\hline Comunicación Intercultural & 2 & 15 & 13.33 \\
\hline T/S Desarrollo municipal & 13 & 23 & 56.52 \\
\hline Total & 752 & 1729 & 38.16 \\
\hline
\end{tabular}

La carrera que con más alto índice de Eficiencia Terminal se destaca es la licenciatura en Enfermería con 82.68 \%. Según la mayoría de las fuentes, ese índice se debe a que en su mayoría son mujeres que ya laboran como enfermeras y optan por sacar la licenciatura, además el registro del título significa una mejora salarial por lo que son los que más agilizan el trámite de titulación.

Comportamiento similar se da con Ciencias de la Educación, que tiene $81.08 \%$ de eficiencia. Una de las razones es que todos y todas son docentes de Educación Primaria y/o Secundaria que imparten clases en los colegios públicos y privados de la Región Autónoma Atlántico Norte. Se valora que como ya están en el campo educativo, son adultos y ya saben a lo que vienen a la universidad, que su título significa mejores oportunidades salariales y de mejores ofertas de trabajo.

En el resto de las carreras baja considerablemente el porcentaje de graduación. La situación cambia en el sentido que no tienen empleos en el campo en el que están estudiando y ha sido notorio que tardan más tiempo en graduarse. En contraste con la carrera de Comunicación Intercultural donde la mayoría de los estudiantes ejercen el trabajo de periodismo, pero apenas el 13.33\% ha logrado graduarse.

\section{Graduación 1995-2005, porcentaje por carreras}

En cuanto al porcentaje de graduación por carrera se destaca Ciencias de la Educación con $81.08 \%$ seguido de Enfermería con $82.68 \%$. En esto tiene que ver la atención brindada y las motivaciones internas y externa de cada egresado o egresadas.

Se constata en este apartado que la carrera con menos índice de Eficiencia Terminal es Comunicación seguida muy de cerca por el Técnico Forestal de Waspam. Se valora que en esta situación ha incidido el hecho de que la coordinación de carrera no estuvo permanente en el recinto y muchas veces se manejó más desde el instituto de 


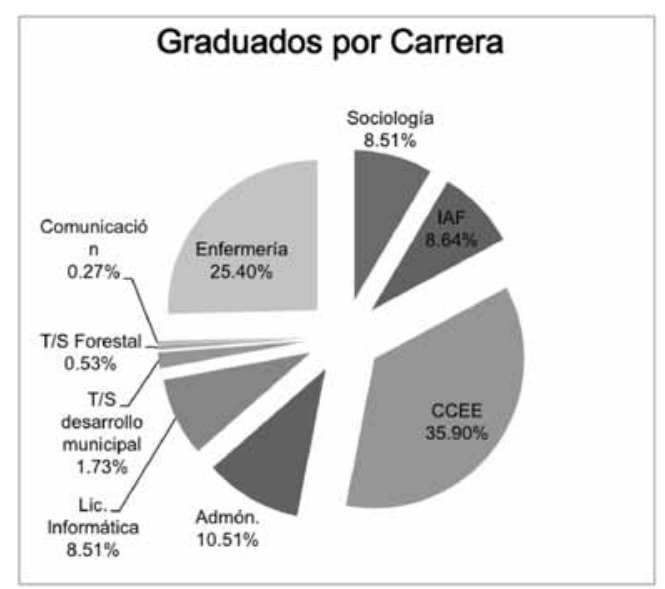

Gráfico No. 2. Porcentaje de graduación por carreras.

comunicación que desde Secretaría Académica que posteriormente se involucra más en la gerencia y atención a la carrera, logrando que los estudiantes culminaran la carrera y quedó casi desatendida en lo que concierne al proceso de graduación, aunque sí se dieron algunas reuniones con quienes egresaron, pero casi nunca se logró juntarlos en su totalidad.

\section{Porcentaje de graduación por etnia}

La mayoría de estudiantes de URACCAN Bilwi son miskitos, le siguen los mestizos, creoles, sumu/ mayangnas y garífunas. En ese mismo orden se refleja el porcentaje de graduados, sólo que en este período no hay graduados de la etnia sumu/mayangna. Los resultados reflejan que debe darse mayor prioridad a la atención de egresados de etnia sumu/mayangna, garífuna y creole.

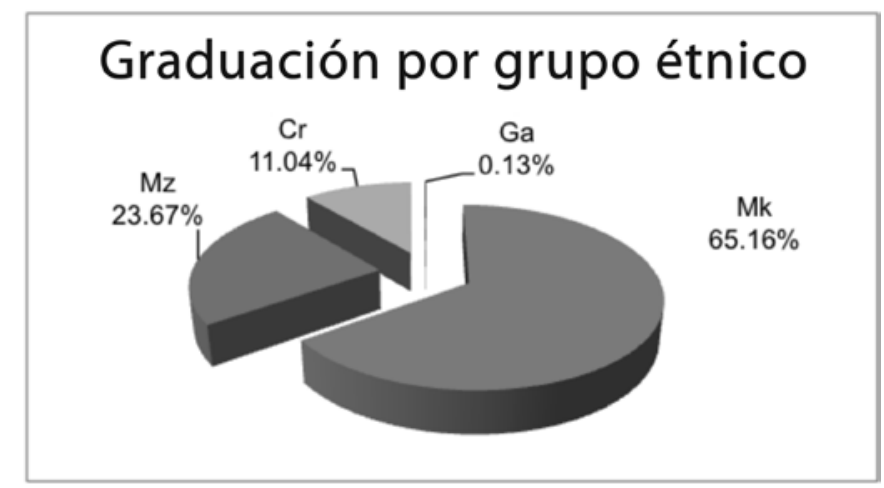

Gráfico No. 3. Porcentaje de graduación por etnia. Mk: miskitu, Mz: mestizos, Cr: Creoles, Ga: Garífunas.

En porcentaje de graduación, el $65 \%$ pertenecen a la etnia miskitu, el $23 \%$ a la etnia mestiza, $11 \%$ creoles y $13 \%$ garífunas. 


\section{Tiempo para graduarse}

La Eficiencia Terminal también fue conceptualizada en la XXX Conferencia Nacional de Ingeniería (2003) como: "la proporción de estudiantes que concluyen un programa en determinado momento, frente al total de los que iniciaron un cierto número de años antes". En este sentido, aunque la proporción de estudiantes que se gradúan no es tan bajo pero tampoco podría decirse que realmente es satisfactorio, por eso debe asumirse como un reto para superarlo.

De acuerdo con el reglamento Documentos Normativos de los Trabajos de Graduación, Capítulo III Del Trabajo Monográfico o Tesis, artículo No. 15: "El / la estudiante dispondrá de un año a partir de la fecha de egreso para realizar el trabajo Monográfico o Tesis y presentar la defensa". Si consideramos lo anterior, de un total de 752 graduados/ graduadas el 58.77\% lo logró en el período reglamentado. El resto se llevó entre 2 y 7 años. Solamente 1 (0.133\%) se graduó en un tiempo de 8 años. 17 lo hicieron entre los 6 y 7 años posteriores a su egreso.

Cuadro No. 2. Duración del proceso de graduación

\begin{tabular}{|l|c|c|c|c|c|c|}
\hline \multicolumn{1}{|c|}{ Carrera } & $\mathbf{0 - 1}$ año & $\mathbf{2 - 3}$ años & $\mathbf{4 - 5}$ años & $\mathbf{6 - 7}$ años & $\mathbf{8 m a ́ s}$ & Total \\
\hline Lic. Sociología & 19 & 36 & 9 & & & 64 \\
\hline IAF & 25 & 28 & 10 & 2 & & 65 \\
\hline CCEE & 28 & 96 & 31 & 14 & & 169 \\
\hline Lic. Admón. & 55 & 23 & 1 & & & 79 \\
\hline Lic. Informática & 43 & 3 & 1 & & & 47 \\
\hline T/S desarrollo municipal & 13 & & & & & 13 \\
\hline T/S Forestal & 4 & & & & & 4 \\
\hline T/S ElB & 101 & & & & & 101 \\
\hline T/S Enfermería & 83 & & & & & 83 \\
\hline T/S Informática & 4 & 12 & & & 1 & 17 \\
\hline Enfermería & 67 & 40 & & 1 & & 108 \\
\hline Lic. Comunicación Intercultural & & & 2 & & & 2 \\
\hline Total & $\mathbf{4 4 2}$ & $\mathbf{2 3 8}$ & $\mathbf{5 4}$ & $\mathbf{1 7}$ & $\mathbf{1}$ & $\mathbf{7 5 2}$ \\
\hline \% & $\mathbf{5 8 . 7 7 7}$ & $\mathbf{3 1 . 6 4 9}$ & $\mathbf{7 . 1 8 1}$ & $\mathbf{2 . 2 6 1}$ & $\mathbf{0 . 1 3 3}$ & \\
\hline
\end{tabular}

Si más de la mitad logró graduarse en el tiempo normado, los demás podrían haberlo logrado también. Es significativo el porcentaje que no logró graduarse en el marco del cumplimiento del reglamento (42\%), eso perjudica los índices de eficiencia de las carreras en que más se tarda el proceso de graduación, por lo que vale la pena el esfuerzo, tarea que deberá tomarse como un reto inmediato. 


\section{Factores relacionados con la Eficiencia Terminal}

Las fuentes encuestadas señalaron diferentes razones por las cuales no han concluido su proceso de graduación. En este sentido se destaca que el $56 \%$ de egresados/ egresadas de URACCAN Recinto Bilwi expresó que no han culminado sus estudios por falta de tiempo.

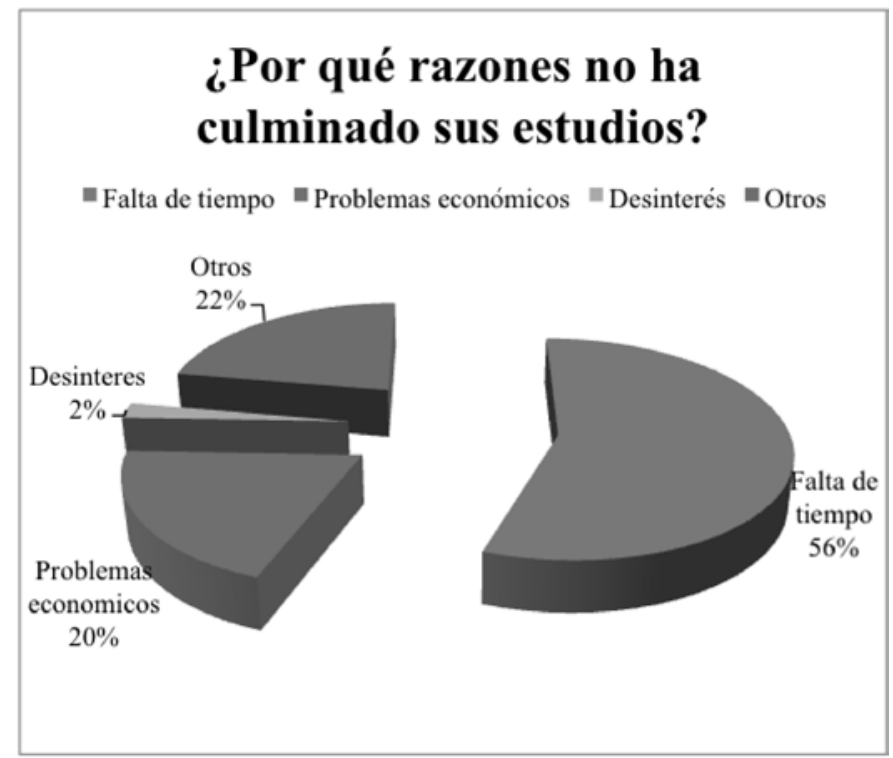

Gráfico No. 4. Porcentaje de problemática relacionada con ET.

Además de esta razón, se mencionaron otras como los problemas económicos y el desinterés. 2 de los egresados mencionaron tener problemas de salud. Se mencionó también que hay algunos problemas con tutores, sienten que no los motivan y que no les dedican tiempo, pero también reconocen que han podido hacer más de lo que hacen para agilizar la conclusión de la etapa de graduación.

Un problema serio, en especial, para las carreras de Ciencias de la Educación y Ciencias de la Salud, es que no tienen habilidad en el manejo de computadoras y eso les vuelve más lento el proceso de graduación; no obstante, lo superan apoyándose en otras personas y muchas veces pagando por ese servicio, lo importante es que sus debilidades o limitaciones las superan y han logrado buenos índices de Eficiencia Terminal en sus respectivas carreras.

\section{Disposición a finalizar estudios}

La mayoría de quienes egresan manifestaron que si bien es cierto que no han podido graduarse, sí les gustaría lograrlo y pronto. 


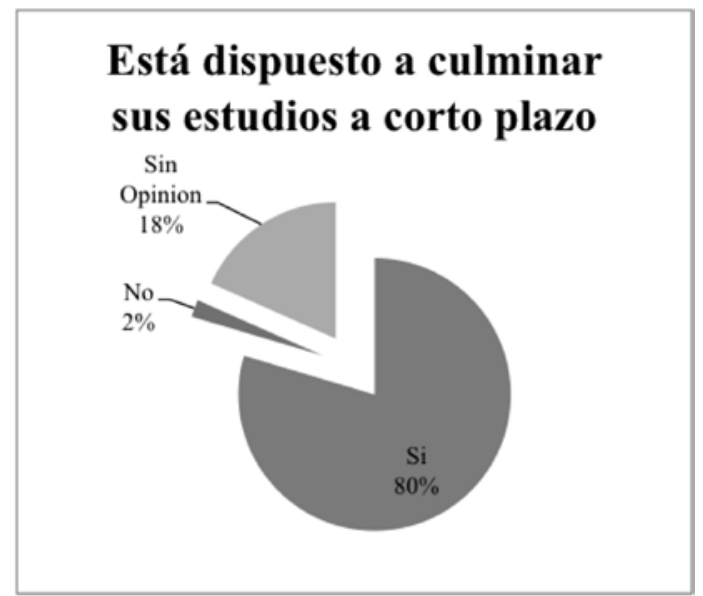

Gráfico No. 5. Porcentaje en disposición de concluir estudios.

A pesar de que la gran mayoría de quienes han egresado dicen querer culminar el proceso de graduación, pero en la práctica se ha demostrado lo contrario en varias carreras.

El 8o \% de los estudiantes egresados, han mostrado interés de culminar sus estudios, sin embargo proponen que las autoridades universitarias realicen mayor motivación y a la vez flexibilización en la aplicación de los reglamentos.

\section{Egresados que no logran graduarse período 1995-2005}

De los egresados de las diferentes carreras que oferta URACCAN Bilwi 876 no han logrado graduarse. La carrera que más egresados tiene es Ciencias de la Educación con 40 estudiantes, el $48 \%$ está en el rango de 2 a 3 años de desfase y el $52 \%$ tienen más de 4 años de ser egresados y no han logrado graduarse.

Los entrevistados de la carrera de Ciencias de la Educación argumentan que no se han graduado por las siguientes razones: falta de tiempo de los tutores, problemas de salud, abandono por parte de compañeros en el momento de la investigación.

Según Rojas (2009:1), uno de los factores que afecta la Eficiencia Terminal señala: "La persistencia e incremento sostenido de la deserción universitaria puede ser, además, síntoma de una profunda crisis social en la valoración de la educación."

\section{Estrategias para elevar la Eficiencia Terminal}

Para dar respuesta a la diversidad de la demanda estudiantil se ha venido implementando diversas modalidades de atención: semestral regular, semestral por encuentros, cuatrimestral y sabatino. Actualmente, ya no se está implementando la modalidad sabatina. 
En los currículos de algunas carreras como informática y administración se han integrado varias formas de culminación de estudios lo que posibilita que egresados y egresadas opten por la que les parezca más motivadora y ágil.

Como parte de las actividades en cada una de las coordinaciones de carrera está la atención a egresados para contribuir a elevar los índices de graduados y brindar atención individual como forma de motivación.

Para dar seguimiento a los egresados/egresadas, en las coordinaciones de carrera se elabora y ejecuta un plan de atención que consiste en actividades como las anteriormente mencionadas: reuniones, asambleas en el último semestre antes que egresen o una vez ya egresados.

Se han dado capacitaciones a tutores, ahora hay más gente que apoya a quienes egresan. Además se han especializado docentes en el campo de la investigación. Con la estrategia de formación profesional postgraduada se han potenciado las investigaciones.

A los tutores y tutoras se les ha ampliado el campo de atención, ahora culminan con sus tutorados hasta que entregan ejemplares, versión digital y el artículo técnico. Con la superación profesional del personal docente se ha potenciado la tutoría, hay mejor manejo de las metodologías de investigación y de la tutoría en sí. Se les da a conocer el reglamento Documentos Normativos de los Trabajos de Graduación y una vez fuera del recinto se les envía cartas, alguna vez se usa la radioemisora para convocar a las reuniones.

Desde las coordinaciones de carrera dan de baja a egresadas y egresados, con previa plática de motivación además una serie de reuniones, llamados, actividades de tutoría y cuando no demuestran interés en graduarse, de manera formal se les envían cartas de ultimátum y al no recibir respuesta a la misiva se les aplica el artículo No. 15 de dicho reglamento, por tanto, no podrá optar al título profesional correspondiente.

\section{Algunas motivaciones para graduarse}

La mayoría del estudiando graduado a quienes se encuestaron, afirmó que lo que más les ayudó a culminar con el proceso de graduación fue su propio interés. Saben que sin el título tienen menos posibilidades de obtención de empleo mejor remunerado.

Coinciden con lo expresado por docentes y coordinadores en cuanto a que el interés de los mismos, un factor determinante para su graduación. Además de su motivación interna, requieren de motivaciones externas para culminar el proceso de graduación. 


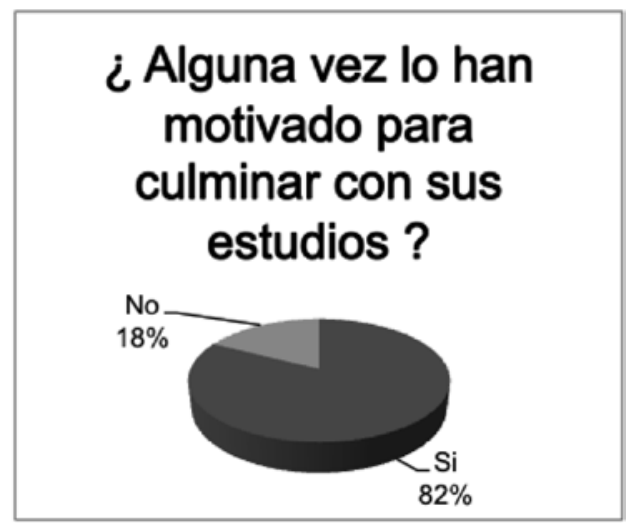

Gráfico No. 6. Porcentaje de interés en concluir estudios.

\section{Apoyo y motivación recibida por egresados/egresadas}

En términos generales puede decirse que sí han recibido apoyo de diferentes instancias. El 36\% expresa que han recibido mucha motivación de algunos familiares, el $25 \%$ ha recibido bastante motivación de sus coordinadores/coordinadoras de carrera, el 21\% ha recibido motivación del personal docente y el 18\% ha recibido apoyo y motivación por parte de sus compañeros/compañeras de clase.

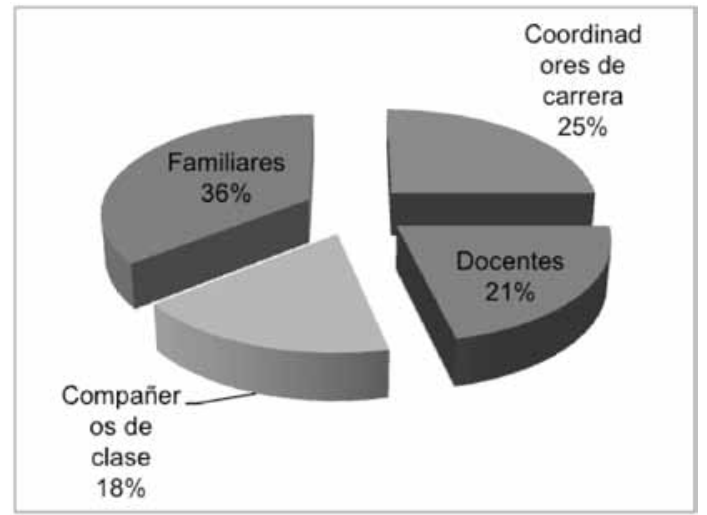

Gráfico No. 7. Apoyo y motivación recibida por los estudiantes, por fuente.

Si sumamos docentes, coordinador y compañeros de clase, todos de la misma carrera, es allí donde está la motivación. Podría pensarse que si en verdad entre todos se hace el esfuerzo planificado e intencionado de apoyar se tendrían mejores niveles de Eficiencia Terminal, Prácticamente de quienes motivan a graduarse, solamente el $36 \%$ es externo a la universidad. 
Desde la coordinaciones de carrera manifestaron que sus instancias han sido determinantes mantener contacto con los egresados a través de reuniones, cartas, llamadas telefónicas y ejercer presión en el cumplimiento del reglamento en el que se estipula que disponen de 6 meses una vez entregado el protocolo para culminar sus monografías.

\section{Propuesta de medidas correctivas para mejorar los índices de Eficiencia Terminal}

Conforme los resultados obtenidos pueden deducirse algunas medidas que podrían ayudar a mejorar la Eficiencia Terminal, como por ejemplo:

- Antes de que concluyan el último cuatrimestre o semestre de estudios cada coordinación de carrera debe entrevistarse o reunirse con los futuros egresados.

- En el currículo debe facilitarse el espacio para que antes de culminar o al egresar ya tengan protocolo aprobado.

- Orientar los pasos que deben dar una vez que hayan egresado.

- Cronogramas de seguimiento consensuados.

- Mayor seguimiento al cumplimiento de los cronogramas.

- Continuar mejorando la atención con las tutorías conjuntas.

- Continuar con las estrategias de formación postgraduada y especialización y potenciar las investigaciones.

- Continuar reflexionando sobre las alternativas de mejora de las tutorías y manejo de metodologías de investigación.

- Es fundamental que la enseñanza de la metodología de la investigación y el seminario monográfico sea teórico y práctico.

- Mantener encuentros sistemáticos entre los tutores y tutoras a fin de conocer problemáticas y logros en las tutorías y ayudar a agilizar los procesos de graduación.

\section{Conclusiones}

La Eficiencia Terminal en este estudio es de $38.16 \%$ definida como la proporción de estudiantes que concluyen un programa, frente al total de los que lo iniciaron. Los estudiantes egresados en el período de estudio de 1995 a 2005, tomando como criterio los que tienen más de un año de atraso, se encontró como resultado de que el 41.22\% 
de los egresados ésta en el rango de dos hasta diez años, el principal argumento que expone el estudiantado es por problemas económicos.

Los estudiantes entrevistados manifestaron que han sido motivados por los docentes y coordinadores de carreras en un $46 \%$, de igual forman han sido motivados por familiares y amigos en $54 \%$.

\section{Recomendaciones}

De acuerdo al diseño de estudio se procuró que las recomendaciones surgieran del grupo meta que a continuación se detalla de manera textual:

1. Evitar enviar cartas desmotivadoras donde notifican pasar los registros por cartas de baja, sin antes conocer los problemas de los estudiantes.

2. Facilitar herramientas de trabajo que agilicen el trabajo a los egresados.

3. Brindar al menos una prórroga de tres meses para trabajar con la monografía.

4. Asignar tutores que realmente tengan tiempo y voluntad para que ayuden a diseñar y seleccionar el tema social de interés para el perfil.

5. Organizar una comisión para brindar seguimiento al estudiantado, ya que muchas veces no saben que libros revisar para su marco teórico.

6. Cumplir con los requisitos y reglamentos de la universidad.

7. Que inmediatamente después de culminar la carrera o durante el último año brinde una asesoría para así poder terminar la monografía.

8. No utilizar términos despectivos a los egresados como por ejemplo: desinteresados, frescos, sin antes conocer la problemática del estudiante.

9. Mantener los correos de los estudiantes para darles seguimiento.

10. Buscar alternativas para hacer trabajo práctico en las instituciones, hacer enlaces con las ONG.

11. Que pongan coordinadores que promuevan a los estudiantes en las distintas ONG.

12. Que el estudiante pueda seleccionar al personal de tutoría y que estos no sean parte de una imposición. Que se pueda trabajar la monografía con grupo de 3, y una vez que culmine el año que se vaya trabajando con el tema.

13. Asignar personas directas que realicen visitas a los estudiantes para conocer la problemática del estudiante.

14. Ofertar cursos de graduación a todas las carreras. 


\section{Lista de referencias}

ANUIES (2000). La educación Superior en el siglo XI. Líneas estratégicas para su desarrollo. Una propuesta de la ANUIES, México, ANUIES.

Camarena R., Chavez A., \& Gómez J. Reflexiones en torno al rendimiento escolar y a la Eficiencia Terminal. Consultado el 13 de abril 2011 de http://www.anuies.mx/ servicios/p_anuies/publicaciones/revsup/reso53/txt2.htm\#4

Cuellar O. \& Bolívar A. (2006). ¿Cómo estimar la Eficiencia Terminal en la educación superior. México. Consultado el 10 de mayo del 20011, tomado de la Web: http:// redalyc.uaemex.mx/redalyc/pdf/604/60413901.pdf

Echemendía. M; García. T; Torres. E; Sánchez. D; Rodríguez. Y; y Torres. A. (2008). La eficiencia académica en la universidad Agraria de la Habana en estrecha vinculación con la orientación ocasional, el ingreso, la permanencia y el egreso. La Habana, Cuba.

Eliézer de los Santos V. Los procesos de permanencia y abandono escolar en educación superior Universidad de Colima, México. http://www.rieoei.org/deloslectores/628Santos. PDF

Rojas (2009). El abandono de los estudios: deserción y decepción de la juventud. Consultado el 04 de mayo 2011, tomado de la web: http://www.cienciared.com.ar/ra/ usr/3/467/hologramatica_n1o_vol4pp75_94.

XXX Conferencia Nacional de Ingeniería (2003). Eficiencia Terminal en los programas de ingeniería. Vol. VI. No. 2. Chihuahua, México. Consultado el 13 de abril 2011, tomado de la web: http://www.google.com/\#sclient=psy\&hl=es\&site=\&sour $\mathrm{ce}=\mathrm{hp} \& \mathrm{q}=\mathrm{estudios}+\mathrm{de}+\mathrm{eficienica}$ terminal $\& \mathrm{aq}=\mathrm{f} \& \mathrm{aqi}=\& \mathrm{aql}=\& \mathrm{oq}=\& \mathrm{pbx}=1 \& \mathrm{f}$ $\mathrm{p}=735861 \mathrm{dbcc} 9 \mathrm{cc}$. 